\title{
New optical power sensors using pyrolytic graphite
}

\author{
Patrick Pinot ${ }^{1, *}$ and Zaccaria Silvestri ${ }^{1}$ \\ ${ }^{1}$ Conservatoire national des arts et métiers, Laboratoire commun de métrologie, 93210 La Plaine Saint-Denis, France
}

\begin{abstract}
We present two experimental configurations based on a repulsion force acting on a piece of pyrolytic carbon $(\mathrm{PyC})$ in a magnetic induction generated by a magnet array to measure a laser power in the range from a few milliwatts to a few watts. The levitation configuration consists in measuring the levitation height change of the $\mathrm{PyC}$ sheet related with the optical power irradiating its surface. The weighing configuration consists in measuring the mass change corresponding to a magnetic repulsion force change acting on a piece of PyC placed on a balance pan and irradiated by a laser beam. The quantities affecting the measurement results have been identified. Examples of measurement results are given. The relative uncertainty of optical power measurement is less than $10 \%$ for the first experimental set-up and around $1 \%$ for the second one. The wavelength dependence on power response of this device has been quantified. The two PyC-based devices presented in this paper offer a new technique for measuring optical power.
\end{abstract}

\section{Introduction}

We present two set-ups using a pyrolytic carbon (PyC) sheet to measure a laser power in the range from $10 \mathrm{~mW}$ to $1 \mathrm{~W}$. These elementary devices are based on a small plate of PyC placed in a magnetic flux density generating a repulsion force on the PyC sheet. This force varies when the sheet is irradiated by a laser beam and is related with the optical power. This leads to an alternative to traditional power meters based on thermal measurement techniques via the Stefan-Boltzmann law and photon-electron interaction.

The first experimental configuration presented in a previous paper [1] consists in using the levitation effect of the PyC sheet above a magnet array. Due to the periodicity of the magnetic flux density, the position of the levitated PyC sheet is steady but its levitation height is modified when the PyC surface is irradiated by a laser beam. This leads to potential energy change:

$$
\Delta E_{t}=E_{0} \frac{\Delta d_{t}}{d_{0}}
$$

where $\Delta d_{t}$ is the levitation height change and $E_{0}$ is the potential energy of the $\mathrm{PyC}$ sheet at the initial levitation height $d_{0}$. The power response in terms of levitation height versus irradiation power is sufficiently linear, sensitive and reproducible to be used as a laser power sensor. The height change $\Delta d_{t}$ depends on the exposure time $t_{\text {exp. }}$. It appears to be a suitable measurement parameter for establishing a relation with the irradiating laser power. Several physical quantities can affect the measurement results. In particular, this levitation configuration is very sensitive to mechanical vibration, draughts and temperature fluctuation.
The second configuration presented in another previous paper [2] consists in detecting the repulsion force change between the PyC sheet and the magnet array. In this case, the PyC sheet is placed on the pan of a mass comparator (Mettler Toledo AX206) and the magnet array is held above the PyC sheet at a short distance. The measurement principle is based on the force change expressed in terms of mass through the mass comparator when the PyC surface is irradiated by a laser beam. Thus, there is an equivalence of the potential energy change and the mass change $\Delta m_{t}$ as follows:

$$
\Delta E_{t}=E_{0} \frac{\Delta m_{t}}{m_{0}}
$$

with the same PyC piece $\left(m_{0}=0.41 \mathrm{~g}\right)$, and for an identical position and fixed distance $\left(d_{0} \approx 0.4 \mathrm{~mm}\right)$ between the PyC surface and the magnet array as in the levitation configuration, we obtain a mass change $\Delta m_{t}$ of about $1 \mathrm{mg}$ in the weighing configuration which is equivalent to a height change $\Delta d_{t}$ of $1 \mu \mathrm{m}$ in the levitation configuration. The weighing configuration is mainly sensitive to the ambient temperature fluctuation, but the linearity and reproducibility are somewhat better in the power range from $0.2 \mathrm{~W}$ to $1 \mathrm{~W}$ in this configuration than in the levitation one. However, energy is dissipated in ambient air, which leads to a slight drift being observed in the mass indication by the mass comparator due to thermal effects. This drift can be easily corrected for.

First results are very encouraging. This confirms the feasibility of new laser power sensors using diamagnetic repulsion which appear interesting for applications in metrology, in industry or in technology development. The need to measure high power lasers accurately in

Corresponding author: patrick.pinot@,cnam.fr

C The Authors, published by EDP Sciences. This is an open access article distributed under the terms of the Creative Commons Attribution License 4.0 (http://creativecommons.org/licenses/by/4.0/). 
real-time in laser manufacturing operations has been highlighted [3].

In this paper, the experimental set-up for the levitation configuration is described in subsection 2.1. The magnetic levitation is presented in subsection 2.2 . Thermal effects are discussed in subsection 2.3 and examples of results presented in subsection 2.4. The second set-up for the weighing configuration is described in subsection 3.1. The buoyancy affecting the weighing is discussed in subsection 3.2. Thermal effects affecting the measurement stability are presented in subsection 3.3 after which examples of measurement results are discussed in subsection 3.4.

\section{Levitation configuration}

\subsection{Experimental set-up}

Figure 1 shows the experimental set-up for the levitation configuration. This device has been improved by the addition of a damping system compared with the first prototype described in a previous paper [1].
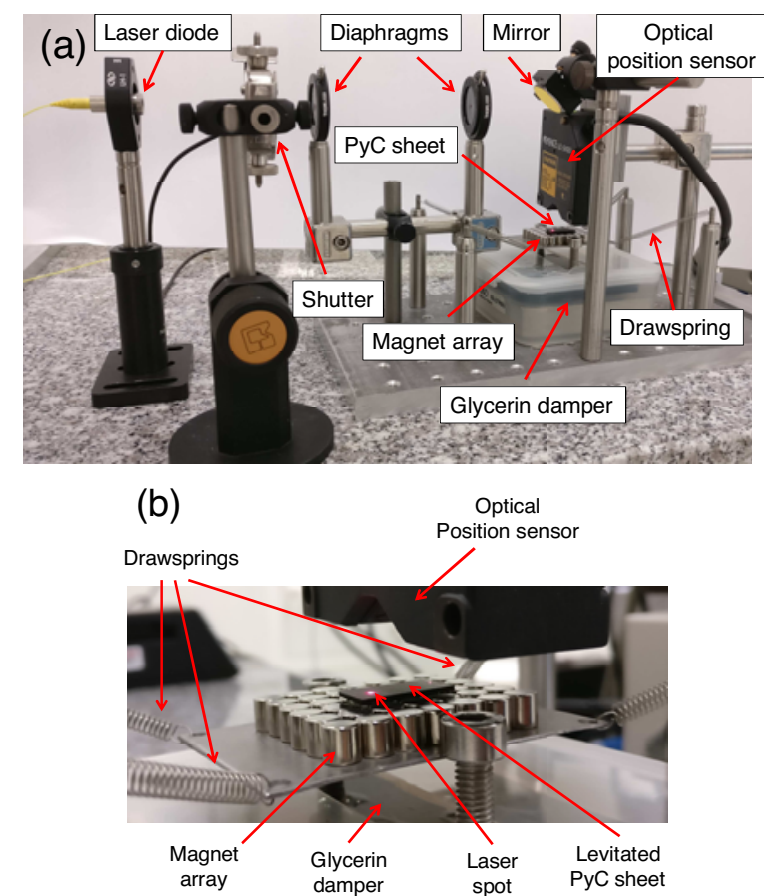

Fig. 1. Photographs of the experimental set-up in the levitation configuration. (a) Overview of the set-up (in this case, the laser under test is a laser diode emitting $3 \mathrm{~mW}$ at $635 \mathrm{~nm}$; (b) Closeup of the magnet array on the damping device and levitated PyC sheet.

The levitation configuration is a very simple device which consists of a magnet array, a sheet of pyrolytic carbon, two diaphragms, a mirror, a shutter and a laser displacement sensor.

The horizontal magnet array is composed of $(5 \times 7)$ cylindrical magnets of diameter and height $6 \mathrm{~mm}$. To attenuate the vibration effect, the magnet array is hung with four springs and is coupled with an horizontal aluminum plate immerged in glycerin to damp the device.

The dimensions of the PyC piece are $24 \mathrm{~mm} \times 8 \mathrm{~mm} \times 1 \mathrm{~mm}$.

The two diaphragms $(\varnothing 1.5 \mathrm{~mm}$ and $1.0 \mathrm{~mm})$ are used to limit the size of the laser spot and to ensure always the same area of the PyC surface is illuminated.

Once the horizontal laser beam has passed through the two diaphragms, it is reflected and directed vertically downwards onto the PyC surface.

The shutter is placed between the laser head and the first diaphragm to control the exposure time.

The laser displacement sensor (Keyence LC-2420) is used to measure the height change of the PyC sheet when its surface is irradiated.

\subsection{Magnetic field}

Several configurations have been studied corresponding to different stable positions of the PyC sheet where its potential energy is the lowest. Given both the sizes of the PyC sheet and of the magnet array, there are only six stable levitation positions. Each PyC position change necessarily entails position changes of the Keyence sensor laser spot and of the irradiation spot of the laser beam under test. Consequently, there is one metrological characterization valid only for one position. Each PyC position is identified as "position $\mathrm{X}$ " where $\mathrm{X}$ is an order number.

Figure 2 shows a photograph of the PyC sheet levitated at about $0.4 \mathrm{~mm}$ above the magnet array surface.

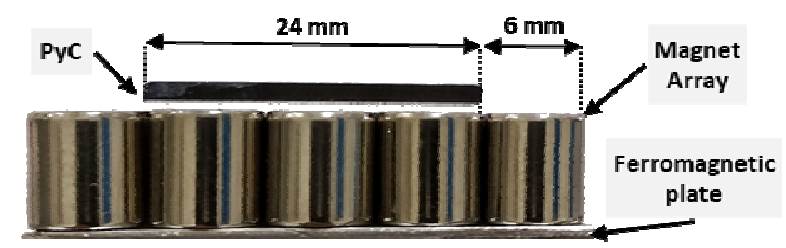

Fig. 2. Photograph of the PyC sheet levitated above the magnet array (position 1).

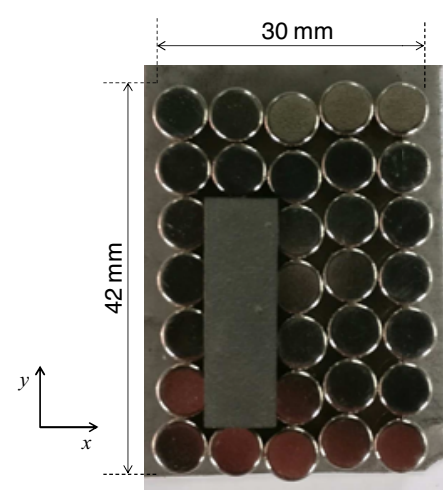

(a)

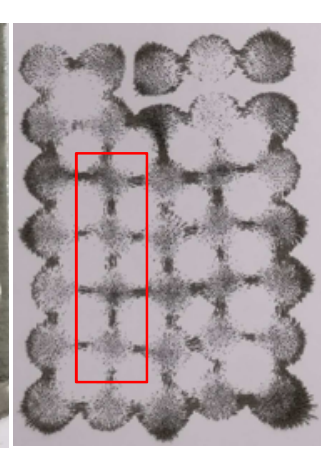

(b)
Fig. 3. Photographs of the magnet array:

a) Arrangement of the $(5 \times 7) 6 \mathrm{~mm}$-diameter magnets with the PyC piece levitated at position 1 ;

b) Same area as shown in Fig 3a covered with a sheet of white paper sprinkled with iron filings. The rectangle represents the $\mathrm{PyC}$ position 1 in this example. 
The photograph of Figure 3.a shows the magnet array with the PyC sheet levitated at one of its stable positions. As expected and shown by the iron filings sprinkled on white paper placed on the magnet array surface in the example of Figure 3.b, the arrangement of the $(5 \times 7) 6$ mm-diameter magnets induces a $2 \mathrm{D}$ periodicity of the magnetic field above the magnet array with a spatial period of about $6 \mathrm{~mm}$.

However, one can see in Fig $3 b$ that the periodicity is not perfect due to misalignments of the magnets. This leads to local heterogeneities of the magnetic field. They can affect the PyC response to an irradiation power according to the levitation position above the magnet array in the horizontal plane.

\subsection{Surface temperature}

To evaluate the risk of any local intense heat and the necessity to limit the exposure time for higher laser powers, thermal tests were carried out using a thermal imaging camera Optris PI (OPTPI16-O31T900).

(a)

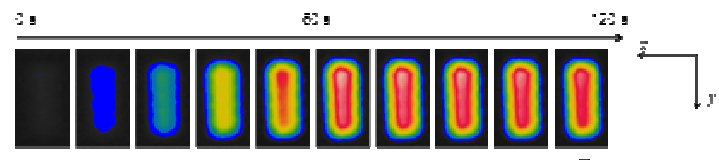

(b)

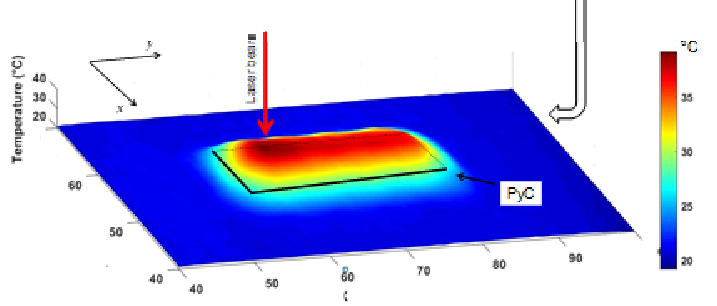

Fig. 4. False color images taken by the thermal imaging camera Optris PI (OPTPI16-O31T900).

a) Sequence of the levitated PyC surface extracted over $120 \mathrm{~s}$ during irradiation by the $1064 \mathrm{~nm}$ laser beam at $0.9 \mathrm{~W}$;

b) Temperature distribution 3D-image after irradiating the PyC surface for $120 \mathrm{~s}$ at a laser power of $0.9 \mathrm{~W}$ (corresponding to the last image of the sequence).

Figure 4.a shows a sequence of successive false color images, taken every $12 \mathrm{~s}$, of the temperature measurement of the PyC surface illuminated by a $1064 \mathrm{~nm}$ laser beam of $0.9 \mathrm{~W}$. The temperature increase seems to tend towards a plateau at about $40{ }^{\circ} \mathrm{C}$ after the surface has been irradiated for $t_{\exp }=120 \mathrm{~s}$. This results from the exceptional thermal properties of this material which presents a high anisotropy. The irradiation induces a photothermal effect which reduces the diamagnetism of the material [4].
Figure 4.b shows a false color image of the PyC surface extracted from a thermal camera recorded after $120 \mathrm{~s}$ of irradiation by the $1064 \mathrm{~nm}$ laser beam at $0.9 \mathrm{~W}$. The temperature imaging threshold was chosen at $22{ }^{\circ} \mathrm{C}$ corresponding to the contour line. The ambient air temperature was $21^{\circ} \mathrm{C}$. The analysis of images taken over $120 \mathrm{~s}$ irradiation shows that the temperature diffusion from the irradiated point at one of the $\mathrm{PyC}$ sheet ends to the other end is not instantaneous. In addition, it seems in Figure 4.b that a temperature increase in the horizontal plane over the PyC edges could correspond either to an imaging artifact (blurring effect) or more probably to a heat exchange with the ambient air close to the $\mathrm{PyC}$ piece.

In other words, this means that there is a heat exchange with the ambient air close to the PyC sheet leading to a balance between absorbed energy (thermally excited electrons by irradiation) and emitted energy (heat transferred to the air).

\subsection{Result and discussion}

Figure 5 shows examples of curves obtained from the recorded data for position 2 for eleven values of irradiation power $P_{i r r}$ at a wavelength of about $1064 \mathrm{~nm}$. The exposure time $t_{\exp }$ was limited to $20 \mathrm{~s}$ and the ambient air temperature was $(22.7 \pm 0.2){ }^{\circ} \mathrm{C}$. These examples are particular because the displacement change versus exposure time is negative and relatively small. This means that the levitation height increases with the exposure time for position 2. In addition, a plateau is reached after the PyC surface has been irradiated for about $10 \mathrm{~s}$. For the other positions, the displacement change is positive and the plateau is ten times higher for the same optical power. Consequently, the device is much more sensitive to the irradiating optical power for the other positions than for position 2 , but the plateau is reached after $60 \mathrm{~s}$ of irradiation instead of $10 \mathrm{~s}$.

Nevertheless, the experimental results showed in Figure 5 can be used to determine the metrological specifications of the PyC-based sensor for position 2 . Figure 6 shows the graphical representation of the levitation height change after an exposure time of $20 \mathrm{~s}$ for the different optical powers in Figure 5. Figure 6 also shows that the photo-response of the sensor is not linear in the range $100-1000 \mathrm{~mW}$. The best fit is obtained by using a second-order polynomial regression. However, the sensor response can be considered linear within two sub-ranges $100-400 \mathrm{~mW}$ and $400-1000 \mathrm{~mW}$. This point is observed for all positions. 

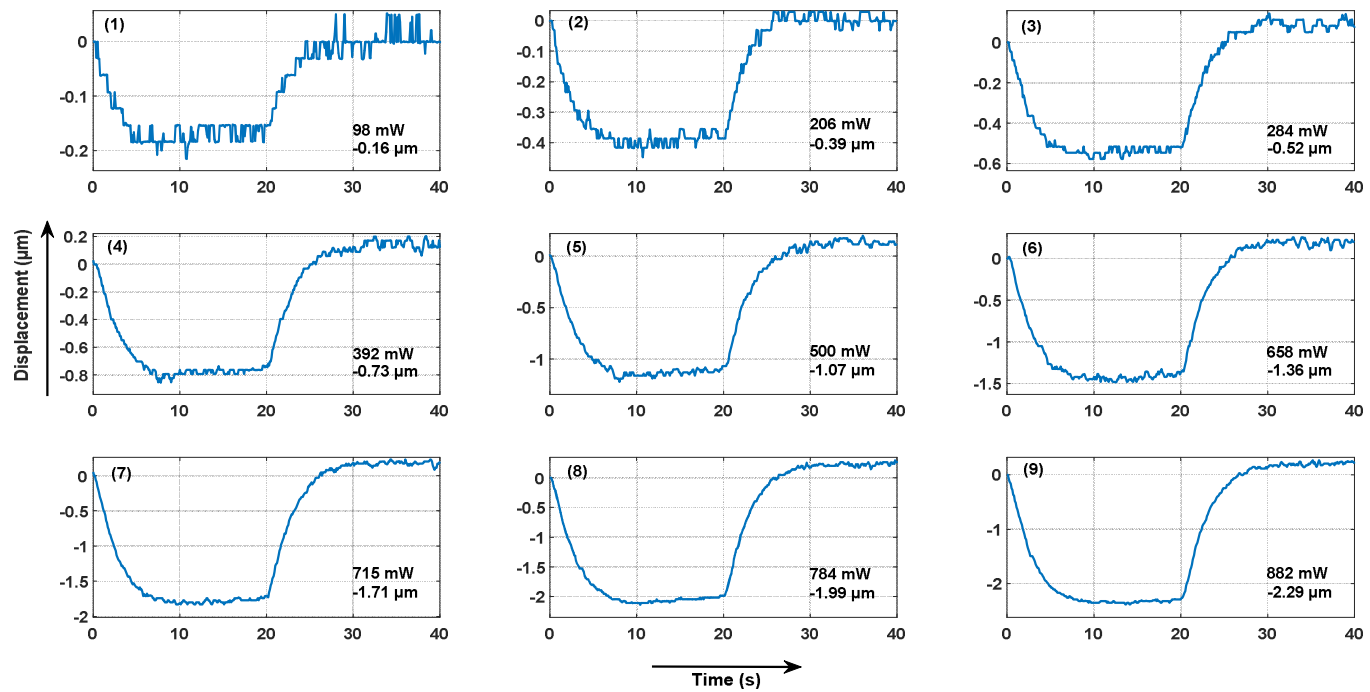

Fig. 6. Examples of curves obtained from experimental data for position 2 of the PyC sheet. The maximum exposure time is $20 \mathrm{~s}$. The irradiation power $\mathrm{P}_{\text {irr }}$ and the maximum differential displacement $\Delta \mathrm{d}_{20}$ are indicated on each graph.
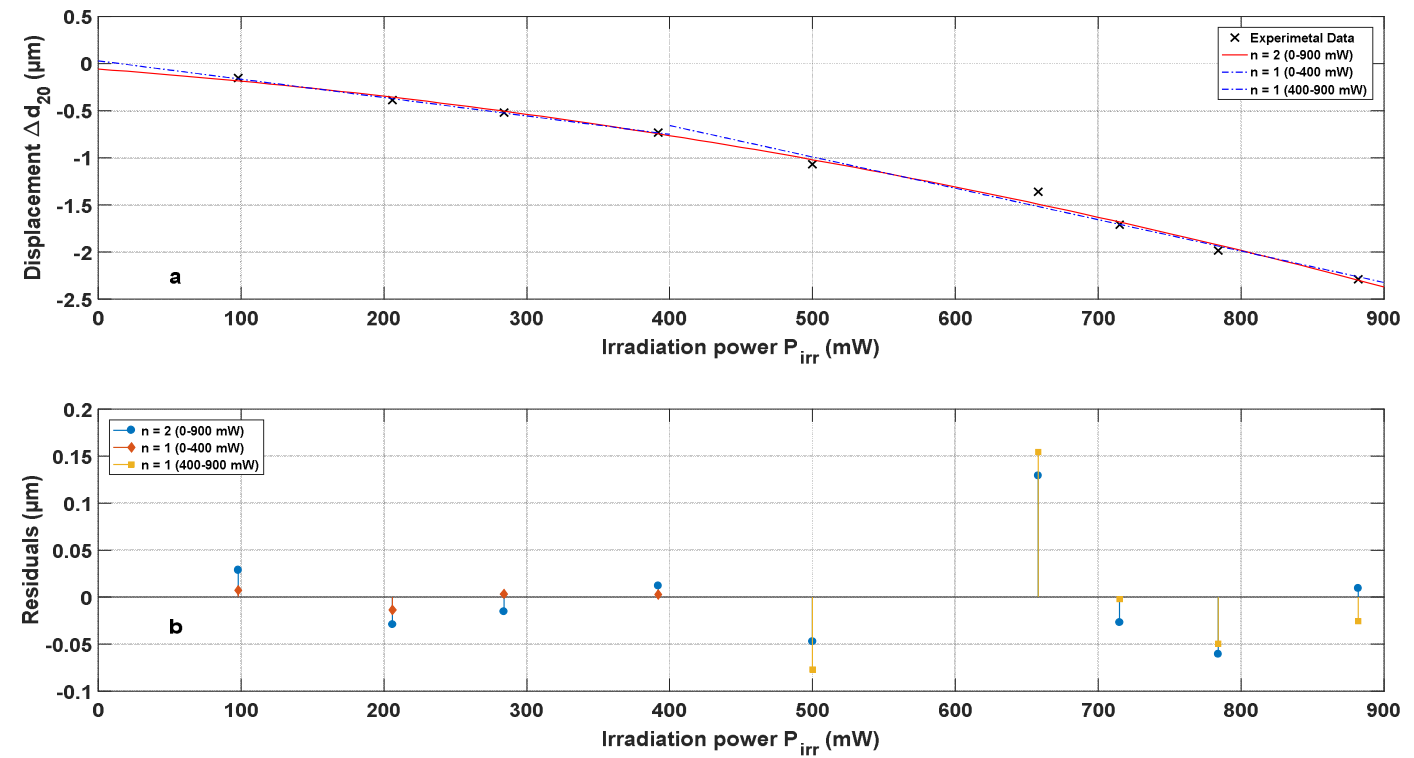

Fig. 5. Graphical representation from experimental data in Figure 5. a) displacement value $\Delta d_{20}$ versus irradiation power $P_{\text {irr }}$; curve from a second-order polynomial fit (solid line) and linear fits (dotted lines); b) residuals for every polynomial fit.

\subsubsection{Background noise}

Note that the sensitivity and the signal-to-noise ratio depend on the PyC configuration. To appreciate this point, just compare the example for two different positions in Figure 7 for $P_{\text {irr }}=46 \mathrm{~mW}$ with an exposure time of $60 \mathrm{~s}$ at $1064 \mathrm{~nm}$. Curves 7a and 7b are obtained for positions 3 and 4 of the $\mathrm{PyC}$ sheet, respectively.

For low power when the height changes either during the irradiation or after closing the shutter are quite low, the background noise is preponderant. There are three main sources of noise, one due to the quantization noise of the Keyence displacement sensor, another due to the airconditioning system and a third one due to ground vibrations. From recordings of $\Delta d_{t}$ when the PyC surface is not irradiated, the standard deviation of the signal is $u_{c r} \approx 0.044 \mu \mathrm{m}$ when the air conditioning system is running while it is $u_{c s} \approx 0.029 \mu \mathrm{m}$ when it is stopped.
Assuming that:

- these values of standard deviation are the quadratic sum of the standard deviations $u_{q}, u_{c}$ and $u_{v}$ respectively associated with the three sources of noise;

- the quantization noise characterized in terms of uncertainty corresponds to the uncertainty associated with resolution following a uniform probability distribution;

we can deduce the following approximate values: $u_{q} \approx$ $0.003 \mu \mathrm{m}, u_{c} \approx 0.033 \mu \mathrm{m}$ and $u_{v} \approx 0.029 \mu \mathrm{m}$. This means that the contribution of quantization noise is negligible compared with the two other sources of which the contribution is almost equivalent. Therefore, all the measurements were carried out without air-conditioning. 

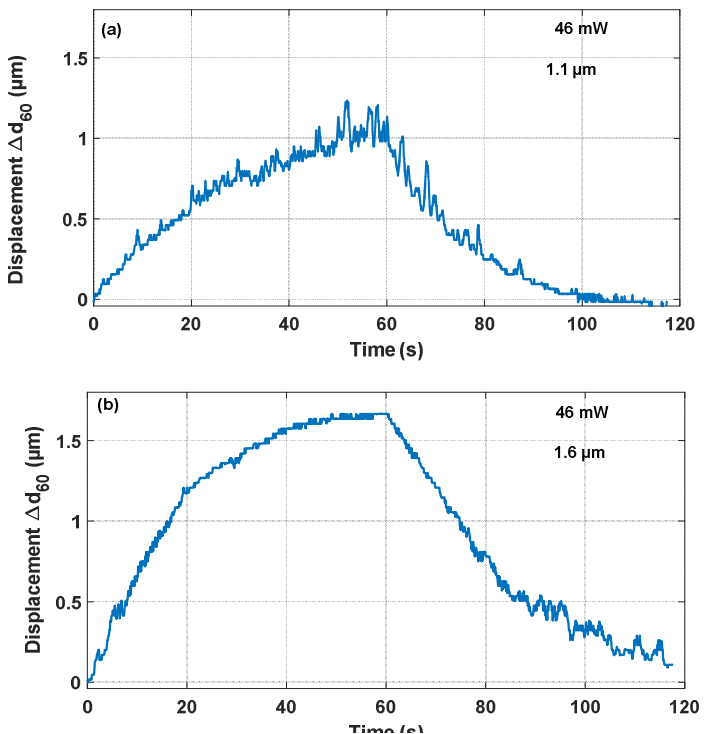

Fig. 7. Curves for two different $\mathrm{PyC}$ positions obtained from experimental data with an exposure time of $60 \mathrm{~s}$ with a 1064 $\mathrm{nm}$ laser beam at $46 \mathrm{~mW}$; (a) position 3; (b) position 4 .

\subsubsection{Sources of error and uncertainties}

In this experiment, there are several sources of error due to parameters affecting the measurement which must be taken into account in the uncertainty budget.

In addition to the uncertainty component due to the repeatability of the $\mathrm{PyC}$ sensor measurements in terms of type A evaluation of uncertainty [5], the main contributions of uncertainty (type B evaluation [5]) for this study come from:

- the power meter which was not calibrated and whose measurement position was not well defined;

- the area of the laser spot and its position on the $\mathrm{PyC}$ surface;

- the background noise due to vibrations;

- the local inhomogeneities of the magnetic field;

- the size and the horizontal position and orientation of the PyC sheet over the magnet array;

- the surface quality and the absorption coefficient of the PyC;

- the position of the displacement Keyence sensor spot on the PyC surface;

- the method in terms of exposure time, sampling time and linearization parameter.

Several contributions of these uncertainty components are made negligible by the use of diaphragms and for a fixed position of the PyC sheet. The sensitivity of the sensor could be improved by choosing the best position and size of the PyC sheet adapted to the magnetic field distribution. In addition, the experiment in this configuration could be greatly improved by protecting the $\mathrm{PyC}$ sheet against ground vibrations and temperature fluctuations to improve the signal-to-noise ratio.

The calibration of this device could consist in determining the calibration coefficient $\beta\left(T, \lambda, t_{\text {exp }}, P\right)$ which depends on the ambient air temperature range $T$, the laser wavelength range $\lambda$, the exposure time $t_{\exp }$ and the laser power range $P$ used in expression (3) linking the laser power $P_{\text {irr }}$ to the levitation height change measured over a short irradiation time $t_{\text {exp }}$ :

$$
P_{\text {irr }}=\beta \Delta d_{t}+\gamma
$$

For instance, with $T=(22.7 \pm 0.2){ }^{\circ} \mathrm{C}, \lambda \approx 1 \mu \mathrm{m}, t_{\mathrm{exp}}=$ $5 \mathrm{~s}$ and $P=[100 \mathrm{~mW} ; 400 \mathrm{~mW}]$, we obtain: $\beta \approx 187 \mathrm{~mW} \cdot \mu \mathrm{m}^{-1}$ and $\gamma \approx 0$ for position 5 .

From these considerations, it seems realistic to be able to develop a laser power sensor using diamagnetic levitation calibrated with a relative measurement uncertainty of few percent or less than $10 \%$ in any case. Note that tests made at $532 \mathrm{~nm}$ seem to show that the PyC photo-response to an irradiation power of this experimental device depends also on the laser wavelength. Other tests made at $635 \mathrm{~nm}$ have shown that the discrimination threshold for the positions given a sensitivity $\beta$ larger than $100 \mathrm{~mW} \cdot \mu \mathrm{m}^{-1}$ is about $3 \mathrm{~mW}$ for an exposure time of around $60 \mathrm{~s}$.

\section{Weighing configuration}

\subsection{Experimental set-up}

Figure 8 shows photographs of the experimental set-up for the weighing configuration.

The device uses the same magnet array, PyC piece, diaphragms, mirror and shutter as those used in the levitation configuration but, in addition, a mass comparator is used to measure the equivalent mass change corresponding to the magnetic interaction force change between the magnet array and the PyC piece when it is irradiated by a laser beam.

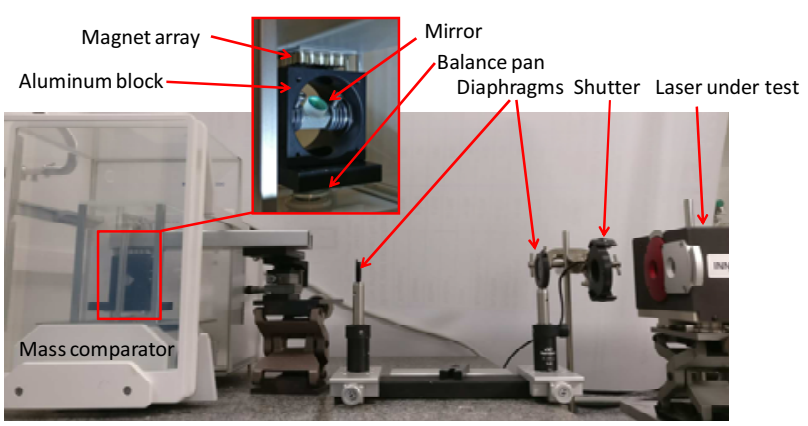

Fig. 8. Photographs of the experimental set-up for the weighing configuration.

In the weighing configuration, the magnet array is horizontally fixed to an aluminum arm linked to a 3D translation stage to adjust its position above the PyC sheet. The PyC sheet piece is glued to an aluminum block placed on the balance pan. The mirror is fixed at $45^{\circ}$ in the aluminum block to reflect vertically the horizontal laser beam onto the PyC surface after it has passed through two diaphragms. 


\subsection{Buoyancy}

The mass $m$ of the device placed on the balance pan is about $200 \mathrm{~g}$ (aluminum block + mirror $+\mathrm{PyC}$ sheet). The main part (98\%) of this mass comes from the aluminum block (density $\rho_{\mathrm{Al}}=2700 \mathrm{~kg} \cdot \mathrm{m}^{-3}$ ). The buoyancy in terms of mass $\Delta m_{a}$ is given by:

$$
\Delta m_{a}=-m \frac{\rho_{a}}{\rho_{\mathrm{Al}}}
$$

where $\rho_{a}$ is the density of moist air.

The buoyancy of the aluminum block is about $-89 \mathrm{mg}$ for an ambient air temperature of about $20{ }^{\circ} \mathrm{C}$ and a conventional air density of $1.2 \mathrm{~kg} \cdot \mathrm{m}^{-3}$.

The density of moist air $\rho_{a}$ can be calculated using the formula recommended by the OIML [6]:

$$
\rho_{a}=\frac{0,34848 p-0,009 H \exp (0,061 T)}{273,15+T}
$$

where $p$ is the pressure expressed in $\mathrm{hPa}, T$ the temperature expressed in ${ }^{\circ} \mathrm{C}$ and $H$ the relative humidity expressed in $\%$.

The relative sensitivity coefficients [6] of the air parameters are as follows:

$$
\begin{aligned}
& \frac{1}{\rho_{a}} \frac{\partial \rho_{a}}{\partial T}=-3.4 \times 10^{-3} \quad \mathrm{~K}^{-1} \\
& \frac{1}{\rho_{a}} \frac{\partial \rho_{a}}{\partial p}=+1 \times 10^{-5} \quad \mathrm{~Pa}^{-1} \\
& \frac{1}{\rho_{a}} \frac{\partial \rho_{a}}{\partial H}=-1 \quad \% .
\end{aligned}
$$

For our device, the measurement indication is not the "absolute" mass, but the mass change over exposure time $t_{\exp }$ or over recording time $t_{\max }$. We choose as criterion that the slope of the buoyancy mass change over one second must be less than $1 \mu \mathrm{g} / \mathrm{s}$. This means that the relative air density change must be less than $1.3 \times 10^{-3}$ for an exposure time of $60 \mathrm{~s}$. In these conditions, the buoyancy change has no significant effect on the mass change measurements. Using expression (6) we obtain the following limits of the variation of ambient conditions for $60 \mathrm{~s}$ exposure:

- $\partial T / \partial t= \pm 0.18^{\circ} \mathrm{C} / \mathrm{min}$;

- $\partial P / \partial t= \pm 0.68 \mathrm{hPa} / \mathrm{min}$;

- $\partial H / \partial t= \pm 7.7 \% / \mathrm{min}$.

Since all the experiments for this study have been carried out in these environmental limits, we need not apply any buoyancy correction.

\subsection{Instability of weighing due to temperature}

In the weighing configuration, as shown in Figure 9, the maximum temperature of the $\mathrm{PyC}$ surface reaches about $25.3^{\circ} \mathrm{C}$ at ambient air temperature of about $21.9^{\circ} \mathrm{C}$ when the $\mathrm{PyC}$ surface is illuminated in the same conditions as that of the levitation configuration. Corrected for the air temperature slope, the temperature change of the $\mathrm{PyC}$ surface is only $3.4^{\circ} \mathrm{C}$. This is very small compared with the $16^{\circ} \mathrm{C}$ measured for the levitation configuration. Two reasons can explain this difference. First, the aperture and the position of the diaphragms D1 and D2 were not exactly the same in the two configurations, while they limit the same optical power irradiating the mirror M1 in the weighing configuration. Secondly, it appears that a significant amount of energy is dissipated through the two contact zones with the $\mathrm{C}$ profile despite the thin insulating sheets. This assumption is confirmed in Figure $9 \mathrm{~b}$ which shows that the two contact zones are at ambient temperature possibly due to conduction heat transfer.

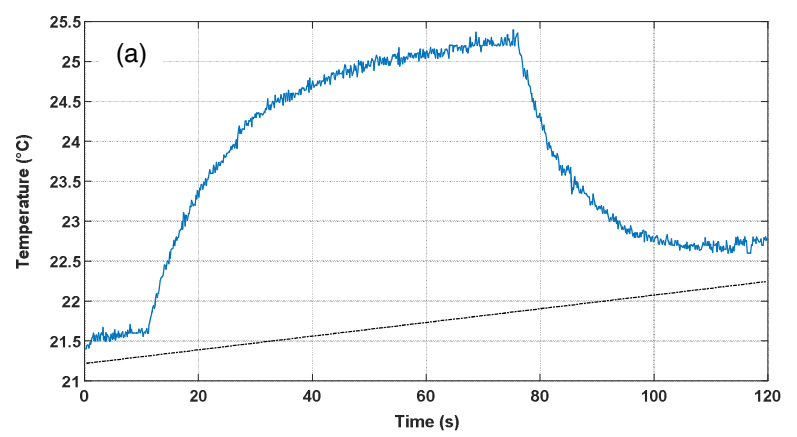

(b)

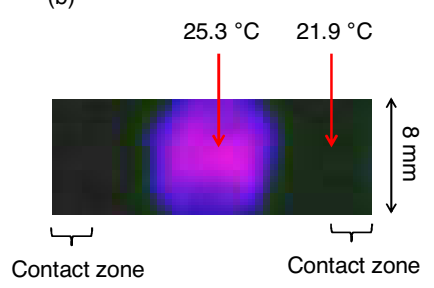

Fig. 9. a) Temperature change of the PyC surface when it is illuminated by a laser beam of wavelength $1064 \mathrm{~nm}$ and optical power $P_{\text {irr }}$ of $0.9 \mathrm{~W}$ for $60 \mathrm{~s}$. Dotted line is the air temperature mean value near the $\mathrm{PyC}$ sheet.

b) False color image of the PyC surface after $60 \mathrm{~s}$ irradiation.

Figure 10 shows the simultaneous measurements without laser irradiation of mass change 10(a) and air temperature close to the pan 10 (b) over $250 \mathrm{~min}$. In order to reduce the influence of air-conditioning system, an enclosure made of transparent plastic has been installed to protect the entire set-up (except the laser) against air movement. The two blue curves 10(a) and 10(b) recorded before the enclosure was installed are clearly correlated. There are large oscillations with a period of about 60 min due to air-conditioning control. The two signals are in opposition of phase. The peak-topeak amplitudes are about $0.5{ }^{\circ} \mathrm{C}$ and $1.8 \mathrm{mg}$ for air temperature and mass changes respectively equivalent to a temperature sensitivity coefficient of $-3.6 \mathrm{mg} \cdot{ }^{\circ} \mathrm{C}^{-1}$. The oscillations are not symmetrical. The maximum positive mean slope (considering the large oscillations) is about $+0.12 \mathrm{mg} / \mathrm{min}$ and the minimum negative mean slope is about $-0.05 \mathrm{mg} / \mathrm{min}$ for the mass change. 

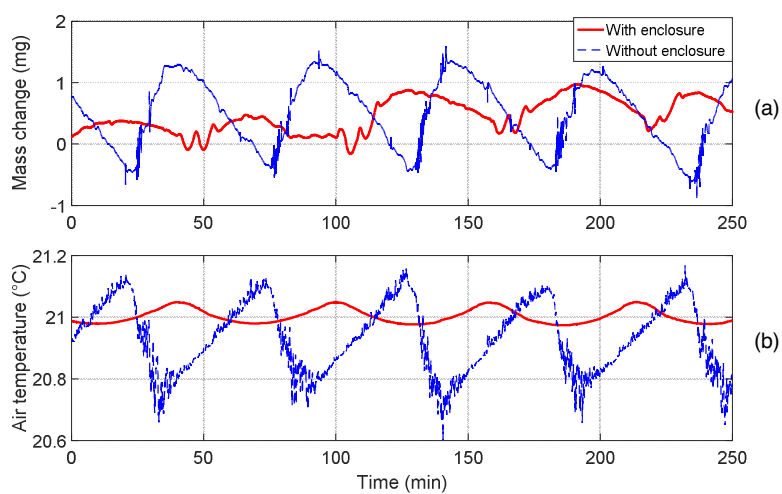

Fig. 10. Measurements without the transparent plastic enclosure (blue curves) and with the enclosure (red curves) over 250 min of: (a) Mass change (without laser irradiation); (b) Ambient air temperature close to the pan.

The amplitude of $1.8 \mathrm{mg}$ observed without the plastic enclosure cannot be explained by the buoyancy change using expressions (4) and (5) giving a temperature sensitivity coefficient of $+0.24 \mathrm{mg} \cdot{ }^{\circ} \mathrm{C}^{-1}$. This effect is of opposite sign and too small compared with the measured temperature sensitivity coefficient.

Another origin might be the magnetic induction variation with temperature. Considering that the manufacturer of $\mathrm{NdFeB}$ magnets gives a typical remanent induction $B_{r}$ of $1.2 \mathrm{~T}$ (independent of the magnet's geometry) and that the temperature coefficient of remanence $\zeta$ is $-0.1 \%{ }^{\circ} \mathrm{C}^{-1}$ for $\mathrm{NdFeB}$ magnets, an approximate calculation has been carried out to verify this assumption.

In our experiment as shown in the previous paper [1], the magnetic flux density in an $x-y$ plane at the height $z$ above the magnet array exhibits a $2 \mathrm{D}$ periodicity due to the magnet array composed of a juxtaposition of small identical permanent magnets in a horizontal plane $x-y$. In this case, the vertical induction component $B_{z}$ has a horizontal periodic and homogeneous distribution. Its mean value in the horizontal plane is constant. In these conditions, a variation of temperature $\partial T$ involves a variation of magnetic induction $\partial B_{r}=B_{r} \zeta \partial T$. This leads to an approximate derivative of magnetic induction related to temperature $\partial B_{r} / \partial T$ of about $-10^{-3} \mathrm{~T} \cdot{ }^{\circ} \mathrm{C}^{-1}$.

We can use the following approximate expression for the magnetic force acting on the PyC sheet:

$$
F_{z} \approx-\chi \cdot \frac{S}{\mu_{o}} \cdot \int_{z} B \frac{\partial B}{\partial z} \cdot d z
$$

where $\mu_{0}$ is the vacuum permeability $\left(4 \pi \times 10^{-7} \mathrm{H} \cdot \mathrm{m}^{-1}\right)$, $\chi$ the volume magnetic susceptibility of pyrolytic carbon $\left(-4 \times 10^{-4}\right)$ and $S$ the base area of the PyC sheet (192 $\left.\mathrm{mm}^{2}\right)$.
The expression to calculate the magnetic flux density $B_{z}$ of a cylinder magnet of diameter and height $2 R$ at the distance $z$ from a pole face on the symmetrical axis is as follows:

$$
B_{z} \approx \frac{B_{r}}{2}\left[\frac{2 R+z}{\sqrt{R^{2}+(2 R+z)^{2}}}-\frac{z}{\sqrt{R^{2}+z^{2}}}\right]
$$

Considering that the levitation force $F_{z}$ acting on the PyC sheet at $\mathrm{z}=d_{0} \approx 0.4 \mathrm{~mm}$ for $m_{0}=0.41 \mathrm{~g}$ is about $4 \mathrm{mN}$ and that expressions (7) and (8) lead to the following expression:

$$
\frac{\partial F_{z}}{\partial T} \approx 2 \frac{F_{z}}{B_{r}} \frac{\partial B_{r}}{\partial T}
$$

we obtain from expression (9): $\partial F_{z} / \partial T=-73 \times 10^{-6} \mathrm{~N} \cdot{ }^{\circ} \mathrm{C}^{-1}$. We deduce an approximate mass variation $\partial m=-3.7 \mathrm{mg}$ for $\partial T=+0.5^{\circ} \mathrm{C}$. Although this result is somewhat twice as large as the measured mass change of $-1.8 \mathrm{mg}$, it corresponds to a temperature sensitivity coefficient $\partial m / \partial T=-7.5 \mathrm{mg} \cdot{ }^{\circ} \mathrm{C}^{-1}$ of the same sign as $-3.6 \mathrm{mg} \cdot{ }^{\circ} \mathrm{C}^{-1}$.

Figure 10 (red curves) shows the efficiency of the plastic protection. The peak-to-peak amplitude of air temperature close to the balance pan is about $0.08{ }^{\circ} \mathrm{C}$ compared with $0.5{ }^{\circ} \mathrm{C}$ without enclosure. One can observe a temperature change period of about $60 \mathrm{~min}$, but the correlation between variations of temperature and mass is much less clear because of other small disturbances of almost the same amplitude (gentle movement of air, vibrations, instability of servocontrol...). Note that the maximum mass oscillation amplitude is about $0.5 \mathrm{mg}$ and that the oscillations between temperature and mass measurements are of opposite phase. In addition, we observe also a double oscillation (period of about $6.5 \mathrm{~min}$ and amplitude of about $0.3 \mathrm{mg}$ ) on the mass change curve after each maximum temperature corresponding to a maximum of the second time derivative of temperature $\mathrm{d}^{2} T / \mathrm{d} t^{2}$ possibly coming from the servo-control system of the mass comparator.

A correction of air temperature based on the linearization of temperature change over $t_{\max }$ has been applied on mass change measurements using a Matlab ${ }^{\circledR}$ program.

\subsection{Results and discussion}

In this configuration, we observe a delay time of several seconds. In order to reduce the measurement time and to take into account the delay time, we choose to determine a response time $\tau$ as the time to reach 1/e of the mass change value at $t_{\exp }$ when $t_{\exp }$ corresponds to the time to reach a quasi-extremum level (quasi plateau). 

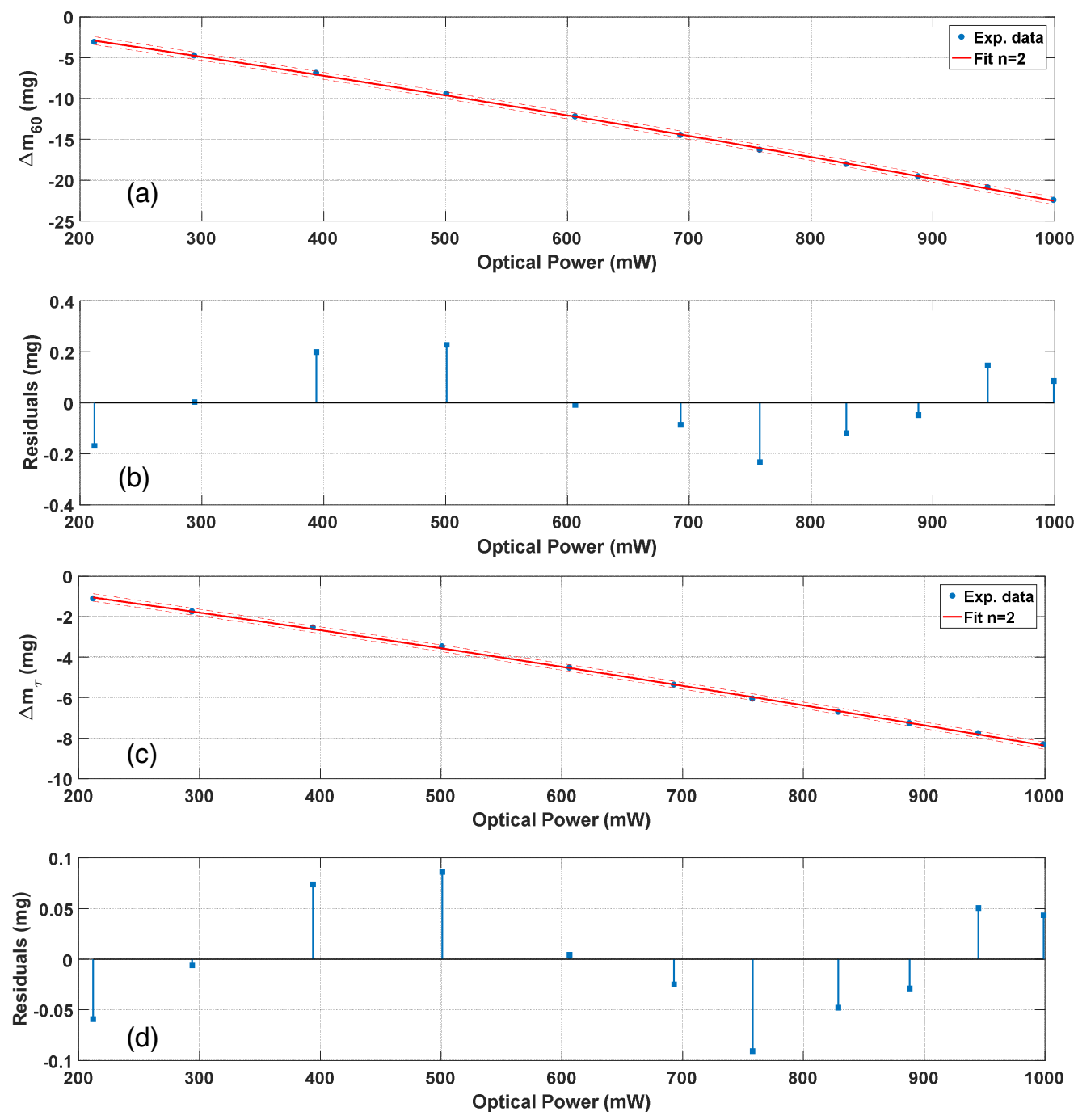

Fig. 11. Mass measurements for four increasing and decreasing (ID) optical power values $P_{\text {irr }}$ between 200 and $1000 \mathrm{~mW}$ generated by the laser of wavelength $1064 \mathrm{~nm}$ : (a) maximum mass changes $\Delta m_{60}$ after a $60 \mathrm{~s}$ exposure time $\left(t_{\exp }\right)$; (b) residuals of $\Delta m_{60}$ for a second-order polynomial fit; (c) mass change $\Delta m_{\tau}$ at $\tau$; (d) residuals of $\Delta \mathrm{m}_{\tau}$ for a second-order polynomial fit.

Figure 11a shows an example obtained from experimental mass changes for four increasing and decreasing optical power values $P_{\text {irr }}$ between 200 and $1000 \mathrm{~mW}$ generated by the laser of wavelength $1064 \mathrm{~nm}$ with an exposure time of $60 \mathrm{~s}$. This example is not one of the best in terms of linearity. Even a second-order polynomial fit gives residuals (Fig 11b) which are not negligible, i.e. about $2 \%$. Figure $11 \mathrm{c}$ shows the mass changes at $\tau$ determined from the measurements in Figure 11a. The response time is about $7.5 \mathrm{~s}$ while the delay time is about $2.5 \mathrm{~s}$. In this case, residuals of the second-order polynomial fit reach about $3 \%$. In better adjustment conditions of the experimental device, we obtained a linearity error less than $\pm 1 \%$ over a sub-range $400 \mathrm{~mW}-1000 \mathrm{~mW}$.

These results show that there is no significant hysteresis phenomenon and that the relative measurement reproducibility is less than $0.4 \%$.
A correction of the temperature drift is applied on experimental mass changes. However, the effect of this correction is weak enough.

We have demonstrated [2] that the relative uncertainty of optical power measurement with this set-up in the range $400 \mathrm{~mW}-1000 \mathrm{~mW}$ at $1064 \mathrm{~nm}$ spreads from $1.6 \%$ to $0.8 \%$ and that the irradiating beam wavelength $(532 \mathrm{~nm}$ and $1064 \mathrm{~nm}$ ) affects the photo-response of pyrolytic carbon.

In addition, Table 1 shows the device is able to measure low optical powers around $20 \mathrm{~mW}$ at $532 \mathrm{~nm}$ for an exposure time of $120 \mathrm{~s}$. The delay time is about $20 \mathrm{~s}$ and the discrimination threshold smaller than $1 \mathrm{~mW}$. 
Table 1. Statistical parameters (mean associated with standard deviation) for three measurement parameters determined from a series of five records for three optical powers $P_{\text {irr }}$ at $532 \mathrm{~nm}$. The differences of the mean values between $15.3 \mathrm{~mW}$ and 17.5 $\mathrm{mW}$ and between $17.5 \mathrm{~mW}$ and $19.5 \mathrm{~mW}$ are determined.

\begin{tabular}{|c|c|c|c|c|}
\hline $\begin{array}{c}\text { Optical power } \\
(\mathrm{mW})\end{array}$ & $\begin{array}{l}\text { Statistical } \\
\text { parameters }\end{array}$ & $\begin{array}{c}\tau \\
(s)\end{array}$ & $\begin{array}{l}\Delta m_{\tau} \\
(\mathrm{mg})\end{array}$ & $\begin{array}{l}\Delta m_{120} \\
(\mathrm{mg})\end{array}$ \\
\hline \multirow{3}{*}{$\begin{array}{l}15.3 \\
\text { (a) }\end{array}$} & mean & 35.86 & -0.2412 & -0.652 \\
\hline & $\begin{array}{c}\text { standard deviation } \\
\text { of mean }\end{array}$ & 0.39 & 0.0061 & 0.016 \\
\hline & $\begin{array}{c}\text { relative standard } \\
\text { deviation (\%) }\end{array}$ & 1.1 & 2.6 & 2.5 \\
\hline \multirow{3}{*}{$\begin{array}{l}17.5 \\
\text { (b) }\end{array}$} & mean & 36.12 & -0.2829 & -0.766 \\
\hline & $\begin{array}{c}\text { standard deviation } \\
\text { of mean }\end{array}$ & 0.25 & 0.0061 & 0.016 \\
\hline & $\begin{array}{c}\text { relative standard } \\
\text { deviation (\%) }\end{array}$ & 0.7 & 2.2 & 2.1 \\
\hline \multirow{3}{*}{$\begin{array}{l}19.5 \\
\text { (c) }\end{array}$} & mean & 36.56 & -0.3261 & -0.8674 \\
\hline & $\begin{array}{c}\text { standard deviation } \\
\text { of mean }\end{array}$ & 0.13 & 0.0024 & 0.00059 \\
\hline & $\begin{array}{c}\text { relative standard } \\
\text { deviation (\%) }\end{array}$ & 0.4 & 0.7 & 0.7 \\
\hline \multirow{3}{*}{$(a-b)$} & mean difference & -0.26 & 0.0417 & 0.114 \\
\hline & standard deviation & 0.46 & 0.0086 & 0.023 \\
\hline & $\begin{array}{c}\text { relative standard } \\
\text { deviation (\%) }\end{array}$ & 177 & 21 & 20 \\
\hline \multirow{3}{*}{$(b-c)$} & mean difference & -0.44 & 0.0432 & 0.101 \\
\hline & standard deviation & 0.28 & 0.0066 & 0.016 \\
\hline & $\begin{array}{c}\text { relative standard } \\
\text { deviation (\%) }\end{array}$ & 64 & 15 & 16 \\
\hline
\end{tabular}

\section{Conclusion}

We have demonstrated that an elementary device consisting of a small plate of pyrolytic graphite levitated above a magnet array is sensitive to a laser power. This leads to an interesting alternative to power meters based either on thermal measurement techniques via the Stefan-Boltzmann law (absorbed energy related to a temperature increase) or on the photon-electron interaction (photodiode effect).

Two different experimental configurations were studied.

The levitation configuration is quite compact but very sensitive to ground vibrations, draughts and ambient temperature changes. The photo-response sensitivity of the experimental set-up is at least $100 \mathrm{~mW} \cdot \mu \mathrm{m}^{-1}$ for most positions of the $\mathrm{PyC}$ sheet, but it is not linear over the whole range 100-1000 $\mathrm{mW}$. Its discrimination threshold is about $3 \mathrm{~mW}$ and the relative measurement uncertainty of optical power in the range $100-1000 \mathrm{~mW}$ at a wavelength of about $1 \mu \mathrm{m}$ is less than $10 \%$.
The weighing configuration is also sensitive to ambient temperature changes. A temperature correction can be applied. In this configuration, there is a delay time of several seconds which is not negligible. The discrimination threshold is smaller than $1 \mathrm{~mW}$. As with the levitation configuration the photo-response is also not linear over the whole range 100-1000 $\mathrm{mW}$. The relative measurement uncertainty of optical power in the range $400-1000 \mathrm{~mW}$ at a wavelength of about $1 \mu \mathrm{m}$ is less than $2 \%$.

First results are very encouraging. This confirms the feasibility of new laser power sensors using diamagnetic repulsion which look promising for applications in metrology, in industry or in technology development.

Many features of these PyC-based devices could be improved. For instance, we are currently studying a new design of PyC-based sensors for industrial lasers of about $1 \mathrm{~kW}$. Its principle consists in splitting off only a few percent of the laser power and using a chopper alternating short irradiation and long darkness periods. Such a device could measure a laser power with a relative uncertainty better than $10 \%$. Although the response time is several seconds, it could be integrated into a real-time system to monitor and control laser powers used in industry. Moreover, in the levitation configuration, a damping system against ground vibrations and a servo-control system of the magnet array temperature using a Peltier module are currently being tested. Ultimately, the best possible improvement would consist in linking the photoresponse to irradiating optical power by a physical law deduced from the optical and electronic properties of PyC. In this case, PyC-based devices could be used as absolute measurement standards.

The authors thank Dr Mark Plimmer for helpful discussions.

\section{References}

1. P. Pinot and Z. Silvestri, New laser power sensor using diamagnetic levitation (submitted in Review of Scientific Instruments)

2. P. Pinot and Z. Silvestri, New laser power sensor using weighing method (submitted in Measurement Science and Technology)

3. P. Williams, B. Simonds, J. Sowards and J. Hadler, Proc. SPIE 9741, High-Power Laser Materials Processing: Lasers, Beam Delivery, Diagnostics, and Applications V, 97410L (2016)

4. M. Kobayashi and J. Abe, J. Am. Chem. Soc, 134, 20593-20596, (2012)

5. JCGM 100:2008, First edition 2008, Corrected version 2010, BIPM.

6. Organisation internationale de métrologie légale (OIML): Weights of classes E1, E2, F1, F2, M1, M1-2, M2, M2-3 and M3: part I. Metrological and technical requirements, International Recommendation OIML R 111, 2004 (Paris). 